\title{
Optimization of some environmental and nutritional conditions using microtiter plate for Pseudomonas aeruginosa biofilm formation
}

\author{
Shaymaa Fouad Rasheed Al-Khazraji | Mohammad Abdul Rahmman Al-Maeni
}

Biology Department-College of Science-Baghdad University, Iraq

Corresponding author: mhogene79@yahoo.com

\begin{abstract}
One of the most important virulence factors in Pseudomonas aeruginosa is biofilm formation, as it works as a barrier for entering antibiotics into the bacterial cell. Different environmental and nutritional conditions were used to optimize biofilm formation using microtitre plate assay by $P$. aeruginosa. The low nutrient level of the medium represented by tryptic soy broth (TSB) was better in biofilm formation than the high nutrient level of the medium with Luria Broth (LB). The optimized condition for biofilm production at room temperature $\left(25^{\circ} \mathrm{C}\right)$ is better than at host temperature $\left(37^{\circ} \mathrm{C}\right)$. Moreover, the staining with $0.1 \%$ crystal violet and reading the biofilm with wavelength 360 are considered essential factors in increasing the productivity of biofilm formation in $P$. aeruginosa. Finally, we highly recommended using these optimized microtitre plate assays to assess biofilm formation in $P$. aeruginosa.
\end{abstract}

Keywords Iraq isolates, $P$. aeruginosa, staining with crystal violet, temperature

\section{Introduction}

$P$. aeruginosa is a Gram-negative bacterium that can cause chronic and fatal infections in immunocompromised individuals, for instance, severe burns or infection by human immunodeficiency virus (HIV) (Whiteley et al 2001). One of the famous bacteria in forming a biofilm is $P$. aeruginosa. This biofilm is important pathogenesis of this bacterium due to the biofilm's role in preventing antibiotics from entering the bacteria (Aybey and Demirkan 2016). This microorganism causes cystic fibrosis due to its ability to form biofilm in the lung causing chronic lung infections (Taylor et al 2014; Maurice et al 2018).

$P$. aeruginosa can also cause nosocomial infections with a chronic state: wound infections, urinary tract infections, and respiratory tract infections. The chronic condition related to disease-causing by $P$. aeruginosa comes from the fact that biofilm formation, a coat of polysaccharide layer, has a role in resisting antibiotic and immune strategies attacks (Taylor et al 2014; Lima et al 2018; Turpin 2015). This correlates with its ability to form a biofilm (Dahmoshi et al 2020).

Biofilm of $P$. aeruginosa is formed from complex microbial communities correlated with matrices that help the bacterium resist desiccation and mechanical removal (Redfern et al 2019). The biofilm consists of matrices formed from complexes of polysaccharides, proteins, extracellular DNA, and lipids (Casciaro et al 2019; Ciofu and Tolker-Nielsen 2019). Polysaccharides mediating biofilm formation in $P$. aeruginosa are alginate, Psl, and Pel. The alginate produced mainly from $P$. aeruginosa lived in cystic fibrosis (CF) patients by the $\operatorname{alg} D$ gene (Kamali et al 2020). Many mechanisms are used to overcome biofilm formation in $P$. aeruginosa, avoiding the interaction between bacteria and surface or destroying biofilm development and maturation (Rasamiravaka et al 2015). Besides, knowledge on biofilm formation's molecular mechanisms may help control chronic infection caused by P. aeruginosa (Mahmmudi et al 2017).

Biofilm inhibitors are considered one factor that affects biofilm formation, such as using glycopeptide dendrimers (Michaud et al 2016). Another factor affecting biofilm formation by $P$. aeruginosa is selective epimerization in the peptide Esculentin-1a (1-21) $\mathrm{NH}_{2}$. (Casciaro et al 2019) and C-Glycosidic LecB Inhibitors (Sommer et al 2019). The importance of biofilm formation is that the bacteria in most of its live form biofilm, while the planktonic single-cell state is considered a transition phase (Ciofu and Tolker-Nielsen 2019). It has been reported by the National Institutes of Health (NIH) that biofilm formation is involved in $65 \%$ of microbial diseases, $85 \%$ of chronic infections, and $90 \%$ of human diseases (Olivares et al 2020; Soler-Arango et al 2019). It has also been reported that the presentence of $P$. aeruginosa for a long time in host tissue correlated with the ability of this bacterium to form a biofilm (Vallet et al 2004). The major risk of $P$. aeruginosa to the patients when causing biofilm on catheters and ventilator tubes is a respiratory infection, particularly ventilator-associated pneumonia (VAP) (Mulcahy et al 2014). In this context, this work's main object was to test different environmental and nutritional conditions for biofilm formation in $P$. aeruginosa bacteria.

\section{Materials and Methods}

\subsection{Bacterial strains}


Eight strains of $P$. aeruginosa were collected from different sources and caused wound infection, urinary tract infection, and respiratory tract infection in different patients. These strains were taken from a master student working on $P$. aeruginosa from different sources. These strains were confirmed as $P$. aeruginosa isolates relying on morphological and biochemical characteristics.

\subsection{Inoculum preparation}

Eight strains of $P$. aeruginosa were activated by culturing them on nutrient broth at $37^{\circ} \mathrm{C}$ for 24 hours, and then these strains were cultured into Luria-Bertani Broth (LB, Difco, USA) at $37^{\circ} \mathrm{C}$ for 24 hours. Centrifugation was carried out at $3000 \mathrm{rpm}$ for $15 \mathrm{~min}$ for cultured $P$. aeruginosa isolates, and the pellet was dissolved in a normal saline solution. After quantification of the bacterial growth, the density of bacteria was adjusted to $10^{9} \mathrm{cells} / \mathrm{ml}$ (Kannan and Gautam 2015; Agarwal et al 2011).

\subsection{Microtiter plate assay}

\subsubsection{Media and incubation}

The formation of biofilm for eight strains of $P$. aeruginosa was investigated in two different broths: LB (Luria Broth) and TSB (Tryptic Soy Broth) to compare the effect of nutritional conditions on biofilm formation.

\subsubsection{Staining}

The staining of biofilm formation was carried out by crystal violet in two different concentrations ( $0.1 \%$ and $0.5 \%$ ) for comparing the effect of environmental conditions on biofilm formation.

\subsubsection{Incubation temperature}

Incubation was achieved at different temperatures 4, 25 , and $37{ }^{\circ} \mathrm{C}$ for comparing the effect of environmental conditions on biofilm formation. This analysis was achieved by incubation in an incubator (BF 56, $59 \mathrm{~L}$ ).

\subsubsection{Wavelength reading}

Reading of OD for biofilm formation was achieved on two different wavelengths, 630 and $405 \mathrm{~nm}$. This analysis was achieved using a spectrophotometer (Cary 100, Varian, Australian).

\subsubsection{Screening the different bacterial strains for biofilm formation}

The screening was carried out on eight strains of $P$. aeruginosa as follows: $20 \mathrm{ML}$ of each $P$. aeruginosa have taken from exponential growth phase and mixed with $180 \mathrm{ML}$ of different broth in the wells of a 96-well microtiter plate and incubated at different temperatures for $48 \mathrm{~h}$. Control was also used, which is the broth without inoculation. Then removing unbound cells was achieved through inverting the plate and vigorous tapping on absorbent paper. After that, the fixation of the cell on the plate was carried out for $30 \mathrm{~min}$ at room temperature. Then the staining of fixed cells was achieved by addition $220 \mathrm{ML}$ of crystal violet (with concentration mentioned above) for $1 \mathrm{~min}$. The stain was then removed by washing with distilled water and dried. In each well, quantification of adherence cells was carried out at wavelengths. Finally, relying on Stepanovic et al (2004), the $P$. aeruginosa strains were classified as weak, moderate, and strong (Kannan and Gautam 2015; Agarwal et al 2011).

\subsection{Statistical analysis}

Statistical test was carried out for the data obtained from the above experiment (Agarwal et al 2011; Stepanović et al 2004). Briefly, cut-off O.D. (O.D.c) was detected and defined as three standard deviations above mean O.D. for the negative control. None biofilm producers, weak, moderate and strong biofilm producer strains were detected as follows; firstly, in the case of O.D. < O.D.c, this means no biofilm producer strains. Secondly, in case of O.D.C < O.D. < or = $(2 *$ O.D.c) this means weak biofilm producer. Thirdly, in case of $(2 *$ O.D.C $)<$ O.D. $<$ or $=(4 *$ O.D.C $)$ this means moderate biofilm producer. Finally, in the case of $\left(4^{*}\right.$ O.D.C) $<$ O.D., this means a strong biofilm producer.

\section{Results}

\subsection{Growth medium}

Production of biofilm using TSB was much higher than using LB. In the TSB medium, most strains were observed to form a biofilm with a strong biofilm producer, while in LB strains were found to be weak and moderate biofilm producers. In TSB, the average OD ranged from $0.05 \pm 0.003$ to $2.7 \pm 0.13$, while in $L B$, the average $O D$ ranged from $0.04 \pm 0.00$ to $1.8 \pm 0.53$ (Figure 1). Therefore, the TSB medium was used to investigate the other factors affecting biofilm production.

\subsection{Staining}

The staining of biofilm using TSB medium with $0.1 \%$ was better than $0.5 \%$ crystal violet. In $0.1 \%$ crystal violet, all strains observed to form a biofilm with strong biofilm producer except one with moderate biofilm producer, while in $0.5 \%$ crystal violet found to be strong, moderate, and weak biofilm producer. In $0.1 \%$ crystal violet, the average OD ranged from $0.05 \pm 0.003$ to $2.7 \pm 0.13$, while in $0.5 \%$ crystal violet, the average $O D$ ranged from $0.06 \pm 0.01$ to $0.8 \pm 0.5$ (Figure 2); therefore, $0.1 \%$ crystal violet was used to investigate the other factors affecting biofilm production.

\subsection{Temperature for biofilm production}

Different temperatures $\left(4,25\right.$, and $\left.37^{\circ} \mathrm{C}\right)$ were used to produce biofilm using TSB medium and crystal violet with $0.1 \%$. There was no significant difference using different temperatures to biofilm production within the eight strains. However, biofilm production was better using room temperature $25^{\circ} \mathrm{C}$, by which all strains observed to form a biofilm with strong biofilm producers (Figure 3). On the other hand, at $4{ }^{\circ} \mathrm{C}$, all strains were found to form a biofilm with 
moderate biofilm producer. At $25{ }^{\circ} \mathrm{C}$, the average $\mathrm{OD}$ was ranged from $0.06 \pm 0.01$ to $2.63 \pm 0.17$. Therefore, the temperature at $25{ }^{\circ} \mathrm{C}$ was used to investigate the other factors affecting biofilm production.

\subsection{Wavelength for biofilm production}

\section{틀 O.D. LB 를.D. TSB}

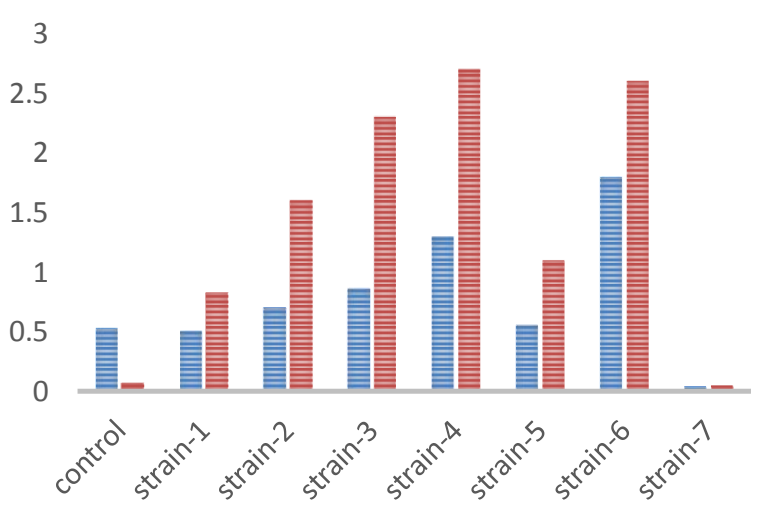

Figure 1 Effect type of broth on biofilm formation in $P$. aeruginosa.

틀 O.D. temperature (4) 틀 O.D. temperature (25)

트 O.D. temperature (37)

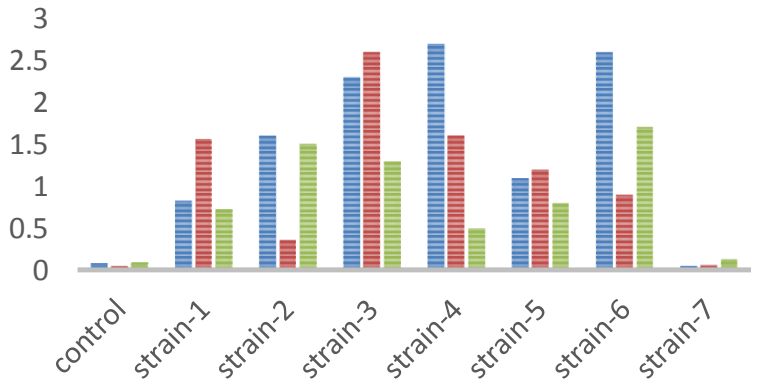

Figure 3 Effect incubation temperature on biofilm formation in $P$. aeruginosa.

\section{Discussion}

Biofilm production using different strains of $P$. aeruginosa is influenced by various factors such as temperature, incubation period, nutrient level, $\mathrm{pH}$, among others. One of the crucial factors affecting biofilm production is nutrient level. In this study, TSB and LB were used to produce biofilm using the eight strains of $P$. aeruginosa; while the TSB considers less rich media, the LB is rich. The results showed that the ODs for biofilm formation using TSB were better than using LB. This result is compatible with Prakash and Krishnappa (2002), who showed that when the bacterial cells grow in low nutrient media, the adhesion of cells will be better; therefore, the amount of biofilm formation is high.

In addition, Gerstel and Römling (2001) state that when the nutrient level is low, the bacteria can express a high amount of aggregative fimbriae, which plays a role in biofilm
Different wavelengths were used to measure biofilm production (630 and $405 \mathrm{~nm}$ ). The different strains produced biofilm using a TSB medium, $0.1 \%$ crystal violet, $25^{\circ} \mathrm{C}$, and reading biofilm at wavelength $630 \mathrm{~nm}$ was better than 405 $\mathrm{nm}$ with average OD ranged from $0.13 \pm 0.03$ to $1.73 \pm 0.03$ (Figure 4).

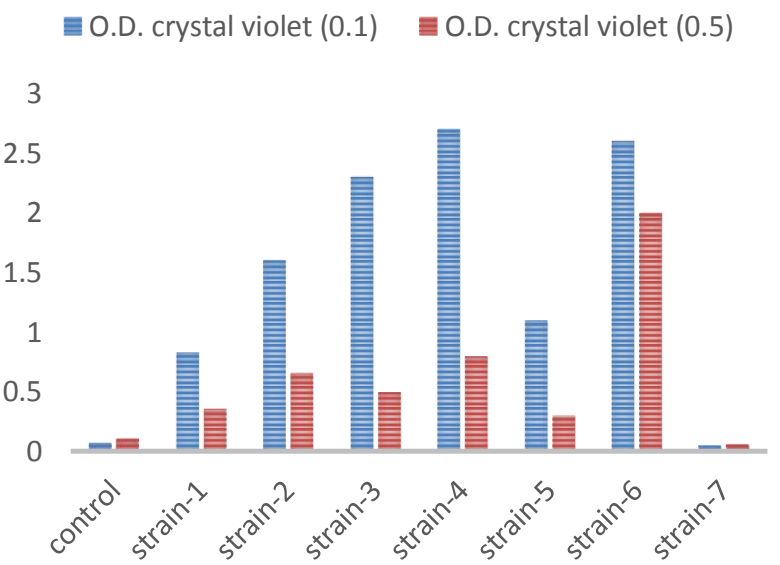

Figure 2 Effect concentration of crystal violet on biofilm formation in $P$. aeruginosa.

$$
\text { 틍.D. wavelength (630) 틀 O.D. wavelength (405) }
$$

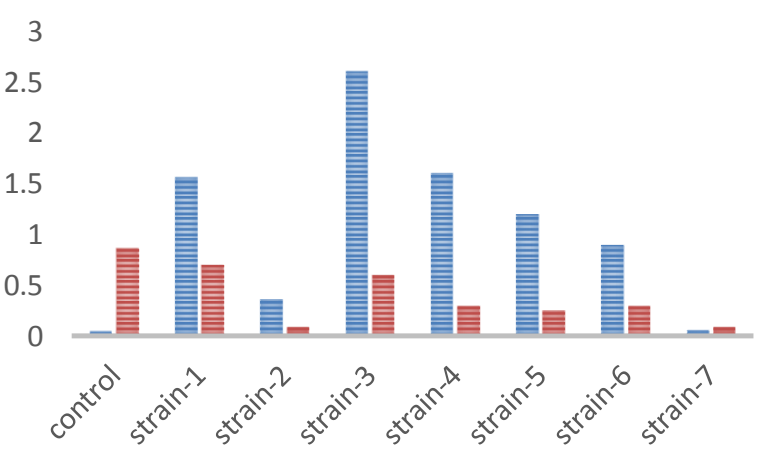

Figure 4 Effect wavelength used in the reading of OD on biofilm formation in $P$. aeruginosa.

formation. The staining of biofilm is another important factor in optimizing the production of biofilm. In this study, staining using $0.1 \%$ of crystal violet was better in biofilm formation than using $0.5 \%$. This result was in contrast with (Stepanović et al 2003) which showed the $0.5 \%$ is better for biofilm formation. The temperature effect on biofilm formation was slightly higher in $25^{\circ} \mathrm{C}$ than $37{ }^{\circ} \mathrm{C}$, and this result was in contrast with Kannan and Gautam (2015), which showed that the biofilm production is higher at $37{ }^{\circ} \mathrm{C}$ due to increased production for polysaccharide matrices by $P$. aeruginosa which constitute the biofilm. It has been reported that the reading of biofilm is mainly taken at $590 \mathrm{~nm}$. However, we use different wavelengths to read the $O D$ of biofilm formation in $P$. aeruginosa. The result was that the reading at the wavelength of $630 \mathrm{~nm}$ was better than $405 \mathrm{~nm}$. This 
result is compatible with Agarwal et al (2011) because 630 $\mathrm{nm}$ is close to $590 \mathrm{~nm}$.

\section{Conclusions}

Different environmental and nutritional factors were used to optimize biofilm production using different strains of $P$. aeruginosa. These factors are represented by the low level of nutrition in the TSB medium and staining with $0.1 \%$ of crystal violet. In addition to the fact that the production was slightly higher at $25^{\circ} \mathrm{C}$ with a wavelength nearby $630 \mathrm{~nm}$. These factors will guide us to minimize the production of biofilm by $P$. aeruginosa in nature.

\section{Conflict of Interest}

The authors declare that they have no conflict of interest.

\section{Funding}

This research did not receive any financial support.

\section{References}

Agarwal R, Singh S, Bhilegaonkar K, Singh V (2011) Optimization of microtitre plate assay for the testing of biofilm formation ability in different Salmonella serotypes. International Food Research Journal 18:1493.

Al-Dahmoshi H, Al-Obaidi RD, Al-Khafaji N (2020) Pseudomonas aeruginosa: Diseases, Biofilm and Antibiotic Resistance. In Pseudomonas aeruginosaBiofilm Formation, Infections and Treatments, IntechOpen.

Aybey A, Demirkan E (2016) Inhibition of Pseudomonas aeruginosa biofilm formation and motilities by human serum paraoxonase (hPON1). AIMS Microbiology 2:388-401.

Casciaro B, Lin Q, Afonin S, Loffredo MR, de Turris V, Middel V, Ulrich AS, Di YP, Mangoni ML (2019) Inhibition of Pseudomonas aeruginosa biofilm formation and expression of virulence genes by selective epimerization in the peptide Esculentin-1a (1-21) $\mathrm{NH}_{2}$. The FEBS journal 286:3874-3891.

Ciofu O, Tolker-Nielsen T (2019) Tolerance and resistance of Pseudomonas aeruginosa biofilms to antimicrobial agents-how $\mathrm{P}$. aeruginosa can escape antibiotics. Frontiers in microbiology 10:913.

Gerstel U, Römling U (2001) Oxygen tension and nutrient starvation are major signals that regulate agfD promoter activity and expression of the multicellular morphotype in Salmonella typhimurium. Environmental microbiology 3:638-648.

Ghaima K, Rasheed S, Ahmed E (2013) Antibiofilm, antibacterial and antioxidant activities of water extract of Calendula officinalis flowers. IJBPR 4:465-470.

Kamali E, Jamali A, Ardebili A, Ezadi F, Mohebbi A (2020) Evaluation of antimicrobial resistance, biofilm forming potential, and the presence of biofilm-related genes among clinical isolates of Pseudomonas aeruginosa. BMC research notes 13:1-6.

Kannan A, Gautam P (2015) A quantitative study on the formation of Pseudomonas aeruginosa biofilm. SpringerPlus 4:1-3.

Khadim M, Marjani M (2019) Pyocyanin and biofilm formation in Pseudomonas aeruginosa isolated from burn infections in Baghdad, Iraq. Biological 12:131.
Lima JLC, Alves LR, Jacomé PRLdA, Bezerra JP, Maciel MAV, Morais MMCd (2018) Biofilm production by clinical isolates of Pseudomonas aeruginosa and structural changes in LasR protein of isolates non biofilm-producing. Brazilian Journal of Infectious Diseases 22:129-136.

Mahmmudi Z, Gorzin A, Branch K (2017) Biofilm of Pseudomonas aeruginosa in Nosocomial Infection. Journal of Molecular Biology Research 7:29-33.

Maurice NM, Bedi B, Sadikot RT (2018) Pseudomonas aeruginosa biofilms: host response and clinical implications in lung infections. American journal of respiratory cell and molecular biology 58:428-439.

Michaud G, Visini R, Bergmann M, Salerno G, Bosco R, Gillon E, Richichi B, Nativi C, Imberty A, Stocker A (2016) Overcoming antibiotic resistance in Pseudomonas aeruginosa biofilms using glycopeptide dendrimers. Chemical science 7:166-182.

Mulcahy LR, Isabella VM, Lewis K (2014) Pseudomonas aeruginosa biofilms in disease. Microbial ecology 68:1-12.

Olivares E, Badel-Berchoux S, Provot C, Prévost G, Bernardi T, Jehl F (2020) Clinical impact of antibiotics for the treatment of Pseudomonas aeruginosa biofilm infections. Frontiers in microbiology 10:2894.

Prakash B, Krishnappa G (2002) Antigenic analysis of outer membrane proteins of biofilm and planktonic cells of Salmonella gallinarum. Indian Veterinary Journal 79:1001-1004.

Rasamiravaka T, Labtani Q, Duez P, El Jaziri M (2015) The formation of biofilms by Pseudomonas aeruginosa: a review of the natural and synthetic compounds interfering with control mechanisms. BioMed Research International 2015.

Redfern J, Wallace J, van Belkum A, Jaillard M, Whittard E, Ragupathy R, Verran J, Kelly P, Enright M (2019) Biofilm Associated Genotypes of Multidrug-Resistant Pseudomonas aeruginosa. bioRxiv: 713453.

Soler-Arango J, Figoli C, Muraca G, Bosch A, Brelles-Mariño G (2019) The Pseudomonas aeruginosa biofilm matrix and cells are drastically impacted by gas discharge plasma treatment: A comprehensive model explaining plasmamediated biofilm eradication. PLoS One 14:e0216817.

Sommer R, Rox K, Wagner S, Hauck D, Henrikus SS, Newsad S, Arnold T, Ryckmans T, Brönstrup M, Imberty A (2019) Anti-biofilm agents against Pseudomonas aeruginosa: a structure-activity relationship study of Cglycosidic LecB inhibitors. Journal of medicinal chemistry 62:9201-9216.

Stepanović S, Ćirković I, Mijač V, Švabić-Vlahović M (2003) Influence of the incubation temperature, atmosphere and dynamic conditions on biofilm formation by Salmonella spp. Food Microbiology 20:339-343.

Stepanović S, Ćirković I, Ranin L, S $\checkmark$ vabić-Vlahović M (2004) Biofilm formation by Salmonella spp. and Listeria monocytogenes on plastic surface. Letters in applied microbiology 38:428-432.

Taylor PK, Yeung AT, Hancock RE (2014) Antibiotic resistance in Pseudomonas aeruginosa biofilms: towards the development of novel antibiofilm therapies. Journal of biotechnology 191:121-130.

Turpin C (2015) The effect of antibiotics on Pseudomonas aeruginosa biofilm production (Doctoral dissertation, Eastern Kentucky University).

Vallet I, Diggle SP, Stacey RE, Cámara M, Ventre I, Lory S, Lazdunski A, Williams P, Filloux A (2004) Biofilm formation in Pseudomonas aeruginosa: fimbrial cup gene clusters are controlled by the transcriptional regulator MvaT. Journal of bacteriology 186: 2880-2890.

Whiteley M, Bangera M, Bumgarner R, Parsek M, Teitzel G, Lory S, Greenberg E (2001) Gene expression in Pseudomonas aeruginosa biofilms. Nature 413:860-864. 